\title{
Succinate dehydrogenase B (SDHB) is differentially expressed in brain metastatic breast cancer.
}

Shahan Mamoor, $\mathrm{MS}^{1}$

1shahanmamoor@gmail.com

East Islip, NY USA

One study has reported a $34 \%$ incidence of central nervous system metastases, including metastasis to the brain, in breast cancer patients treated with trastuzumab ${ }^{1}$. We mined published microarray data ${ }^{2,3}$ to discover genes associated with brain metastasis in breast cancer. We identified significant differential expression of the mitochondrial complex II subunit succinate dehydrogenase B, encoded by SDHB 4,5 , in the brain metastases of patients with metastatic breast cancer. SDHB may be relevant to the biology underlying colonization of the brain with metastatic breast cancer clones.

Keyword: breast cancer, metastasis, brain metastasis, central nervous system metastasis, SDHB, succinate dehydrogenase B, systems biology of breast cancer, targeted therapeutics in breast cancer. 
One study reported a 34\% incidence of central nervous system metastases in patients with breast cancer treated with the monoclonal antibody trastuzumab (Herceptin) ${ }^{1}$. Treatment options for patients with brain metastatic breast cancer are limited ${ }^{6}$. We mined published microarray datasets ${ }^{2,3}$ to understand in an unbiased and systems-level fashion genes whose expression most significantly distinguished brain metastases from primary tumors of the breast, the cells from which these metastatic clones are presumably derived. We found significant differential expression of SDHB in the brain metastases of patients with brain metastatic breast cancer.

\section{Methods}

We utilized microarray datasets GSE $125989^{2}$ and GSE1005343 for this differential gene expression analysis of brain metastatic breast cancer in conjunction with GEO2R. GSE100534 was generated using Affymetrix Human Gene 1.0 ST Array technology with $n=16$ primary tumors from patients with breast cancer and $n=3$ brain metastases from patients with brain metastatic breast cancer. GSE43837 was generated using Affymetrix Human X3P Array technology with $n=19$ primary breast tumors and $n=19$ brain metastases from patients with breast cancer, analysis performed using platform GPL1352. The Benjamini and Hochberg method of $p$-value adjustment was used for ranking of differential expression but raw $p$-values were used to assess statistical significance of global differential expression. Log-transformation of data was auto-detected, and the NCBI generated category of platform annotation was used. A statistical test was performed to evaluate whether SDHB gene expression was significantly between primary breast tumors and brain metastases in humans with breast cancer using a two-tailed, unpaired t-test with Welch's correction. We used PRISM for all statistical analyses of differential gene expression in human breast cancer (Version 8.4.0)(455).

\section{Results} breast cancer.

We mined published microarray data ${ }^{2,3}$ to discover genes associated with brain metastasis in SDHB is differentially expressed in brain metastatic breast cancer.

We identified succinate dehydrogenase B, encoded by SDHB, as among the genes whose expression changes most significantly when comparing brain metastases to primary tumors of the breast in patients with brain metastatic breast cancer ${ }^{2}$ (Table 1$)$. When sorting each of the genes expressed in brain metastases based on significance of change as compared to primary tumors of the breast, SDHB ranked 76 out of 22277 total transcripts (Table 1), equating to $99.7 \%$ differential expression. Differential expression of SDHB in brain metastases in brain metastatic breast cancer was statistically significant (Table $1 ; p=1.88 \mathrm{E}-04)$.

We queried a second microarray dataset ${ }^{3}$ to validate transcriptome-wide differential expression of SDHB in brain metastatic breast cancer. We again found that SDHB was among the genes whose expression changed most significantly when comparing metastases of the brain to primary tumors of the breast in patients with breast cancer (Table 2). When sorting each of the genes expressed in brain metastases based on significance of change as compared to primary tumors of the breast, SDHB ranked 2088 out of 33297 total transcripts (Table 2), equating to $93.7 \%$ differential expression. Differential expression of SDHB in brain metastases in brain metastatic breast cancer was statistically significant (Table $2 ; p=1.64 \mathrm{E}-03$ ). 
SDHB is expressed at significantly lower levels in brain metastases as compared to primary tumors of the breast in brain metastatic breast cancer.

We obtained exact mRNA expression values for SDHB from brain metastases and primary tumor of the breast to evaluate magnitude and direction of change in SDHB expression in brain metastases in patients with breast cancer. SDHB was expressed at lower levels in brain metastases in patients with breast cancer and this difference was statistically significant (Figure $1 ; p=0.0001$ ). We calculated a mean fold change of $0.60 \pm 0.17$ in SDHB expression when comparing brain metastatic tissues to primary tumors of the breast (Table 1).

Thus, we found that SDHB was among the genes, globally, whose expression was most different when comparing primary tumors of the breast to brain metastases in patients with breast cancer, and present at significantly lower quantities at the mRNA level in brain metastatic tissues as compared to primary tumors of the breast.

\section{Discussion}

During cellular respiration, electron transport is performed by five complexes: Complex I-IV. Complex II of the mitochondria consists of four succinate dehydrogenase subunits: SdhA, SdhB, SdhC and $\mathrm{SdhD}$, and effects transfer of electrons to ubiquinone, and functions in oxidation of succinate to produce fumarate; this involves Fe-S clusters in $\mathrm{SdhB}^{4,5}$. In nematodes, the mev-1 mutants are deficient in their ability to catalyze transport of electrons from succinate to ubiquinone; mev-l is an allele of succinate dehydrogenase cytochrome b560, or $\mathrm{SdhB}^{5}$. Under conditions of high oxygen $(60 \%)$, the lifespan of mev-1 mutants is markedly decreased as compared to wild-type N2 nematodes ${ }^{5}$. Mutation of SDHB is found in patients with familial pheochromocytoma and in familial paraganglioma, a disease featuring vascular tumors of the head and neck ${ }^{7}$. Mutation of SDHB is associated with paraganglioma syndrome type 4 (PGL-4), while mutation of SDHD is associated with paraganglioma syndrome type 1 (PGL-1); while 11/32 patients with SDHB mutation possessed malignant disease including pheochromocytoma and paraganglioma, 0/34 patients with SDHD mutation possessed malignant disease ${ }^{8}$. All 11 patients with SDHB mutation possessed distant metastases to the lung or bones ${ }^{8}$. Thus, mutation of SDHB is specifically associated with tumorigenesis and metastasis in humans. One study described suppression of SDHB expression in breast cancer cells by TGF- $\beta$ produced by tumor associated macrophages, supporting tumor growth and angiogenesis through increased glycolysis ${ }^{9}$.

We report here that the gene encoding succinate dehydrogenase B, SDHB, was among those whose expression was most quantitatively different in metastases to the brain when compared to primary tumors of the breast in patients with metastatic breast cancer. SDHB may be of relevance to the biology underlying any number of steps important for metastasis to the brain: exit from the breast, traversing the vasculature and the blood-brain barrier, and colonization of the brain. 


\section{References}

1. Bendell, J.C., Domchek, S.M., Burstein, H.J., Harris, L., Younger, J., Kuter, I., Bunnell, C., Rue, M., Gelman, R. and Winer, E., 2003. Central nervous system metastases in women who receive trastuzumab-based therapy for metastatic breast carcinoma. Cancer, 97(12), pp.2972-2977.

2. Iwamoto, T., Niikura, N., Ogiya, R., Yasojima, H., Watanabe, K.I., Kanbayashi, C., Tsuneizumi, M., Matsui, A., Fujisawa, T., Iwasa, T. and Shien, T., 2019. Distinct gene expression profiles between primary breast cancers and brain metastases from pair-matched samples. Scientific reports, 9(1), pp. $1-8$.

3. Schulten, H.J., Bangash, M., Karim, S., Dallol, A., Hussein, D., Merdad, A., Al-Thoubaity, F.K., AlMaghrabi, J., Jamal, A., Al-Ghamdi, F. and Choudhry, H., 2017. Comprehensive molecular biomarker identification in breast cancer brain metastases. Journal of translational medicine, 15(1), p.269.

4. Guzy, R.D., Sharma, B., Bell, E., Chandel, N.S. and Schumacker, P.T., 2008. Loss of the SdhB, but Not the SdhA, subunit of complex II triggers reactive oxygen species-dependent hypoxia-inducible factor activation and tumorigenesis. Molecular and cellular biology, 28(2), pp.718-731.

5. Ishii, N., Fujii, M., Hartman, P.S., Tsuda, M., Yasuda, K., Senoo-Matsuda, N., Yanase, S., Ayusawa, D. and Suzuki, K., 1998. A mutation in succinate dehydrogenase cytochrome b causes oxidative stress and ageing in nematodes. Nature, 394(6694), pp.694-697.

6. Robson, M., Im, S.A., Senkus, E., Xu, B., Domchek, S.M., Masuda, N., Delaloge, S., Li, W., Tung, N., Armstrong, A. and Wu, W., 2017. Olaparib for metastatic breast cancer in patients with a germline BRCA mutation. New England Journal of Medicine, 377(6), pp.523-533.

7. Astuti, D., Latif, F., Dallol, A., Dahia, P.L., Douglas, F., George, E., Sköldberg, F., Husebye, E.S., Eng, C. and Maher, E.R., 2001. Gene mutations in the succinate dehydrogenase subunit SDHB cause susceptibility to familial pheochromocytoma and to familial paraganglioma. The American Journal of Human Genetics, 69(1), pp.49-54.

8. Neumann, H.P., Pawlu, C., Pęczkowska, M., Bausch, B., McWhinney, S.R., Muresan, M., Buchta, M., Franke, G., Klisch, J., Bley, T.A. and Hoegerle, S., 2004. Distinct clinical features of paraganglioma syndromes associated with SDHB and SDHD gene mutations. Jama, 292(8), pp.943-951.

9. Gómez, V., Eykyn, T.R., Mustapha, R., Flores-Borja, F., Male, V., Barber, P.R., Patsialou, A., Green, R., Panagaki, F., Li, C.W. and Fruhwirth, G.O., 2020. Breast cancer-associated macrophages promote tumorigenesis by suppressing succinate dehydrogenase in tumor cells. Science signaling, 13(652).

PAGE 4 
PAGE 5 
PAGE 6 


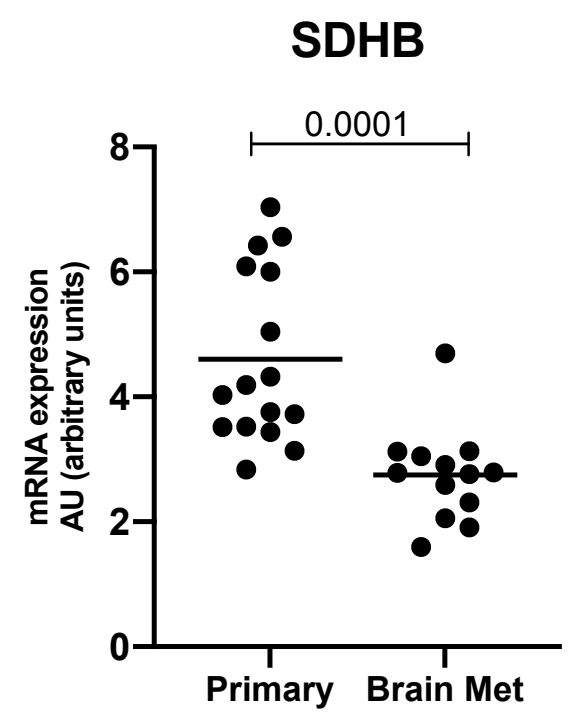

Figure 1: SDHB is expressed at significantly lower levels in brain metastases as compared to primary tumors of the breast in patients with brain metastatic breast cancer.

The mRNA expression level of SDHB in primary tumors of the breast (left) and in the brain metastases of brain metastatic breast cancer patients (right) is graphically represented here with mean SDHB levels marked and the result of a statistical test evaluating difference in mRNA expression level between brain metastases and primary tumors of the breast, a $p$-value, listed above. 